\title{
Sensitization of the Crayfish Lateral Giant Escape Reaction
}

\author{
Franklin B. Krasne and David L. Glanzman' \\ Department of Psychology and Brain Research Institute, University of California, Los Angeles, California 90024
}

Most behavioral reactions that habituate can also be dishabituated by strong stimuli. In the best studied cases, dishabituation seems to be the result of an independent "sensitization" of the behavioral reaction that compensates for habituation without necessarily abolishing it. Crayfish lateral giant (LG) neuronmediated escape reactions are one of the most fully analyzed behavioral reactions that are prone to habituation; however, sensitization/dishabituation of LG escape has not previously been reported.

Here, the effect of strong AC shocks to head or abdomen on the ability of 0.1 msec "test" shocks to sensory roots innervating the tailfan to elicit an LG escape response was examined. Following single AC shocks, test shock threshold for cliciting LG escape reliably fell $5-80 \%$ and recovered over $15 \mathrm{~min}$ to 1 hr. When AC shocks and test shocks alternated at 90 sec intervals, test shock threshold rapidly dropped to an asymptote that was maintained as long as AC shocks were given (up to $2 \mathrm{hr}$ ); following such repeated $\mathrm{AC}$ shocks, recovery often required a number of hours but was complete within 24. Comparable sensitization is seen in the response of interneuron $A$, the largest of a set of sensory interneurons that links afferents to LGs. AC shocks (to either head or tail) no longer sensitize abdominal LG reflex circuitry if the nerve cord is severed between thorax and abdomen. Thus, sensitization appears to depend on a neurally conducted influence that arises in the rostral half of the animal. Pharmacological evidence suggests that octopamine may mediate the sensitization.

Most behavioral reactions that habituate can also be enhanced in excitability, or sensitized, by presenting strong or novel stimuli (Groves and Thompson, 1970; Thompson and Spencer, 1966). From an adaptive point of view this sensitization can serve to heighten reactiveness to potential threats in novel or hostile environments and restore responsiveness to stimuli previously rendered ineffective by habituation (dishabituation).

Such sensitization has been particularly well analyzed in the Aplysia gill and siphon withdrawal reflex. There, the location of the neural change responsible seems to be largely or entirely at the presynaptic side of synapses between relevant sensory neurons and their followers, which is also the location of change responsible for habituation of the reflex (see Kandel and Schwartz, 1982). The change responsible for sensitization is believed to be induced by input to the terminal from a presynaptic "facilitator" neuron that releases either a serotonin-like molecule or a peptide when sensitizing stimuli are presented. This presynaptic facilitatory input increases the amount of transmitter re-

Received Mar. 7, 1985; revised July 24, 1985; accepted Oct. 3, 1985

This research was supported by United States Public Health Service Grant NS 08108. We thank Tina Brown, Gretchen Garrett, Terri Teshiba, Tania Bentler, David Cho, and David Anisman for help in running experiments, and Carl Higgins for typing the manuscript.

Correspondence should be addressed to Franklin Krasne.

'Present address: Center for Neurobiology, Howard Hughes Medical Institute, 722 W. 168th Street, New York, NY 10032

Copyright (C) 1986 Society for Neuroscience $0270-6474 / 86 / 041013-08 \$ 02.00 / 0$ leased by sensory neuron spikes by virtue of producing a persistent decrease in the $\mathrm{K}^{+}$currents that repolarize the terminal after a spike (Kandel and Schwartz, 1982; Klein et al., 1980). Recently, evidence has been obtained suggesting that identical effects, in exaggerated form, mediate classical conditioning of the reflex (Carew et al., 1981, 1983; Hawkins et al., 1983; Walters and Byrne, 1983). This possibility gives new importance to the phenomenon of sensitization and generates a need to determine the extent to which the findings regarding locus and mechanism of sensitization as well as those connecting sensitization to classical conditioning have generality.

A system that holds particular promise for probing the generality of the findings from Aplysia is the lateral giant fiber (LG) mediated escape reaction of the crayfish. The reaction habituates (Wine et al., 1975), the circuitry mediating the reaction is well analyzed (Krasne, 1969; Zucker, 1972a, b), and, though the circuit mediating the reaction is more complex than that found to mediate defensive withdrawal in Aplysia, the change responsible for habituation is, as in Aplysia, a use-produced reduction of transmitter release from sensory neuron terminals (Krasne, 1976; Zucker, 1972b). However, neither sensitization nor any form of dishabituation has been reported in this system, and few signs of its existence have been seen during extensive behavioral and physiological investigations of the reflex (Krasne, unpublished observations; R. S. Zucker, personal communication).

An attempt to obtain sensitization of the LG escape reaction at this time was prompted by the above considerations and was encouraged by the recent discovery that exogenous application of octopamine, a naturally occurring monoamine of the crayfish nervous system, increases excitability of LG reactions (Glanzman and Krasne, 1983). It was also hoped that these experiments would lead to the discovery of circumstances under which an endogenous octopaminergic facilitatory system might operate. To maximize the chances of finding the phenomenon, the experiments were done on free, intact animals with chronically implanted stimulating and recording electrodes.

\section{Materials and Methods}

Animals

Procambarus clarkii about $2.5 \mathrm{~cm}$ rostrum-telson were used in all experiments.

\section{Preparation of animals}

Recording and stimulating electrodes were "skewers" made from 00 stainless steel insect pins (Wards) insulated by Epoxylite except for a $0.5 \mathrm{~mm}$ segment in the middle of the pin. To implant these electrodes the thorax was swaddled with wet cloth and the animal tied down ventral side up. Electrodes for recording ventral cord activity were inserted into a small hole in one pleural plate, thrust through the soft belly skin near the lateral margin of the abdomen, passed under or over the nerve cord, which could be seen through the soft belly skin, thrust out through the contralateral skin and passed through a small hole in the contralateral pleural plate. The skewers were then adjusted so that the uninsulated region was in contact with the nerve cord (Fig. 1, $A_{1}$ and $A_{2}$ ). In experiments where we were primarily interested in firing of LGs, the skewer 


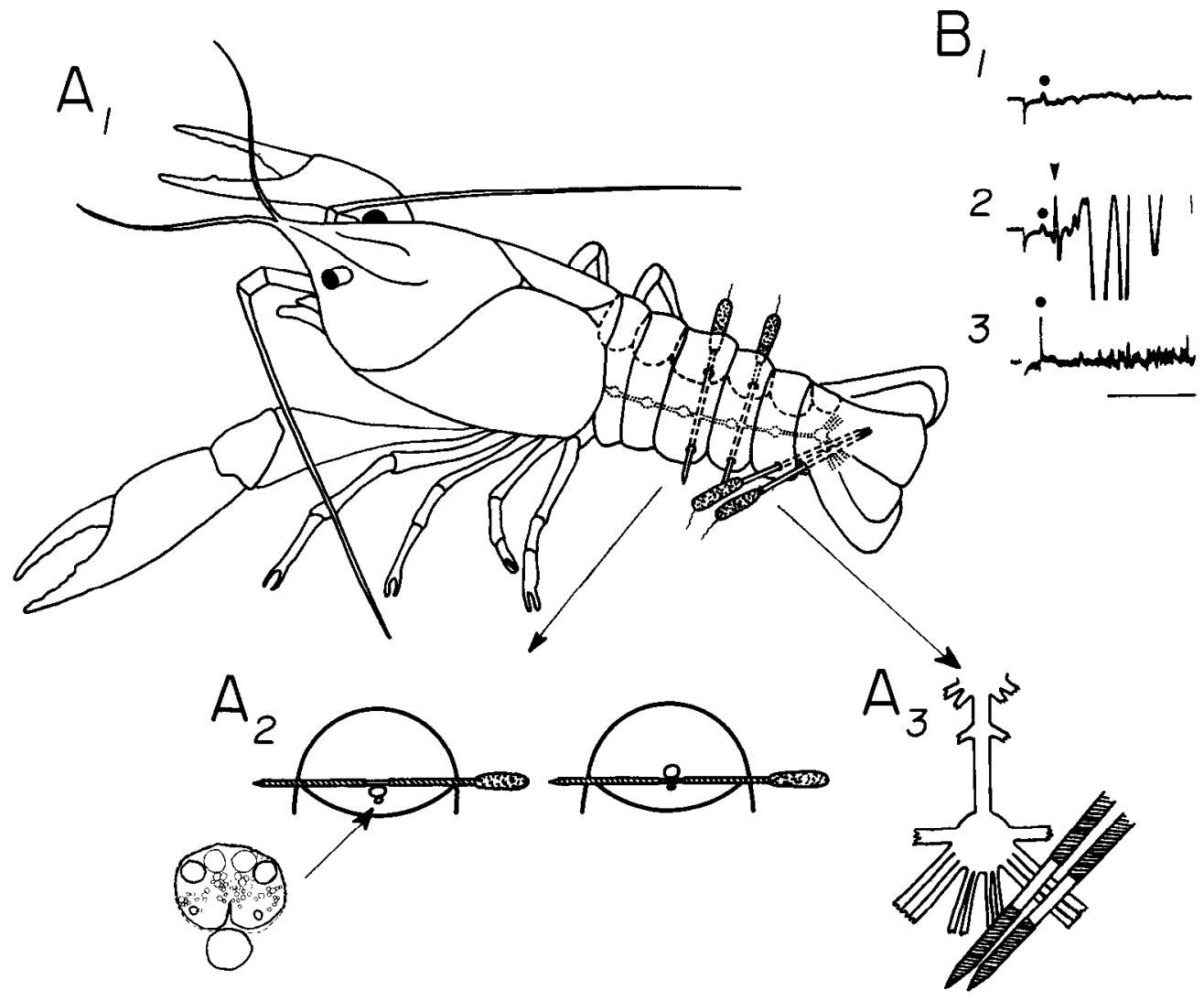

Figure 1. Preparation and sample traces. $A$, The preparation $\left(A_{t}\right)$ showing location of recording and test stimuli electrodes (detailed in $A_{2}$ and $A_{3}$, respectively). $A_{2}$, The position of skewe electrodes used for optimal recording of giant axons (left) and interneuron $A$ (right). Inset in $A_{2}$, The relative sizes and locations in the nerve cord of the LG (the giant dorsolateral axons) and interneuron As (the large, ventrally located axons); the ventral artery is shown to scale underneath. $B$, Typical responses recorded to test stimuli. In each trace, the first deflection is stimulus artifact. $B_{1}$ and $B_{2}$, Dots mark interneuron A spikes, the triangle the LG spikes, as seen by a dorsaily placed skewer. Marked unit in $B_{3}$, An interneuron A spike as seen by a ventrally placed skewer. Time calibration, $5 \mathrm{msec}$.

was passed dorsal to the cord. When we were more interested in monitoring interneuron $A$, the largest of the first-order sensory interneurons in the LG escape reaction circuit, we passed the skewer ventrally between cord and ventral artery. However, either type of placement usually allowed firing of both LGs and interreuron A to be recognized. Skewer electrodes were placed in adjacent segments (usually lying between ganglia 4 and 5 and ganglia 3 and 4) for recording large connective units differentially against an aquarium ground electrode.

Stimulating electrodes were skewers (Fig. $1, A_{1}$ and $A_{3}$ ) placed so as to lie against roots 2,3 , and 4 of the last abdominal ganglion. Prior to electrode implantation, the ipsilateral tailfan and more caudal intersegmental joints were immobilized with a cyanoacrylate glue (Histoacryl blue) to prevent flexion of the caudal part of the body or uropod movements that would alter the position of nerves being stimulated. The more rostral electrode was then inserted into a hole in the fifth pleural plate on the side to be stimulated, slid rostromedially into the body cavity and under the last sternal rib so as to lie over or under roots 2 , 3 , and 4 , where they intersected the caudal edge of the rib in their posterolateral course to the periphery, and thrust out through the soft skin of the opposite side. The more caudal anode was inserted in the sixth pleural plate, thence into the body cavity via a hole placed laterally in the last sternal rib and pushed out of the body through the soft skin of the opposite side. Electrode leads were anchored to the dorsal side of abdominal segment 2 and passed to a float, where connections to electronics were made as necessary for experimental sessions.

For giving large AC shocks (see below), a pair of platinum or copper wires with $2 \mathrm{~mm}$ uninsulated at their ends were usually glued to the animal so that one electrode lay just in front of the rostrum and the other $5 \mathrm{~mm}$ in front of that. When caudal AC shocks were wanted, a similar pair of electrodes was glued to the dorsal surface of the tailfan contralateral to the internally implanted stimulating skewers. In the initial experiments, AC shock electrodes were glued to a wand and brought near the desired part of the animal by hand.

After implantation, animals were kept individually in well-aerated 5 gallon aquaria. At least $1 \mathrm{~d}$ was allowed for recovery before testing began. Effective stimulating and recording could be carried out indefinitely in these preparations. We kept most for several weeks to a month.

\section{Stimulation and recording}

Test stimuli were $0.1 \mathrm{msec}$ negative pulses isolated from ground by a transformer. Stimulus levels are reported here in arbitrary units, but a level of 1.0 is approximately $4.5 \mathrm{~V}$ at the stimulating electrodes.

LG reactions were unambiguously recognized by large LG spikes at recording electrodes followed by large muscle potentials (Fig. $1 B$ ). The latency of LG firing was usually within about $4 \mathrm{msec}$, but occasionally longer. We used a $20 \mathrm{msec}$ oscilloscope sweep; any LG firings occurring at greater than $20 \mathrm{msec}$ were scored as nonresponses even though behavioral responses (some of which were long-latency LG reactions and some of which were non-giant-mediated tail flips) occurred.

Interneuron A (see Kennedy, 1971; Sigvardt et al., 1982) was defined as the first unit with a spike easily discriminable from noise to fire reliably at less than $6 \mathrm{msec}$ latency in an ascending stimulus series. Its latency, except with just-suprathreshold stimuli, was ordinarily about 2-3 msec (Fig. 1, $B_{3}$ and $B_{1}$ ). These characteristics were shown in independent experiments to positively identify a unit whose conduction velocity and receptive field were unique to interneuron $A$.

Sensitization producing stimuli were $35 \mathrm{~V}$ AC shocks $1-10 \mathrm{sec}$ in duration (traumatic shock, or TS). In preliminary experiments (those using a single TS; see Results), these were always of $10 \mathrm{sec}$ duration and were delivered by passing current between a distant indifferent electrode and a $2 \mathrm{~mm}$ diameter, $5 \mathrm{~mm}$ long segment of stainless steel rod mounted on a wand held near the desired part of the animal. The animal was chased with the electrified wand for the desired duration of the shock. In later experiments (those using multiple TSs; see Results), shocks were delivered via electrodes glued to the rostrum or tailfan as described above; usually we tried $5 \mathrm{sec}$ duration shocks in initial TS sessions, but often used other durations in later sessions in order to get preliminary information on the effect of duration.

\section{Experimental procedures}

One session 1-3 hr long was run per day. Electronic apparatus was attached to connectors on floats a half-hour before the start of a session. In most cases (exceptions noted in context), test stimuli through im- 

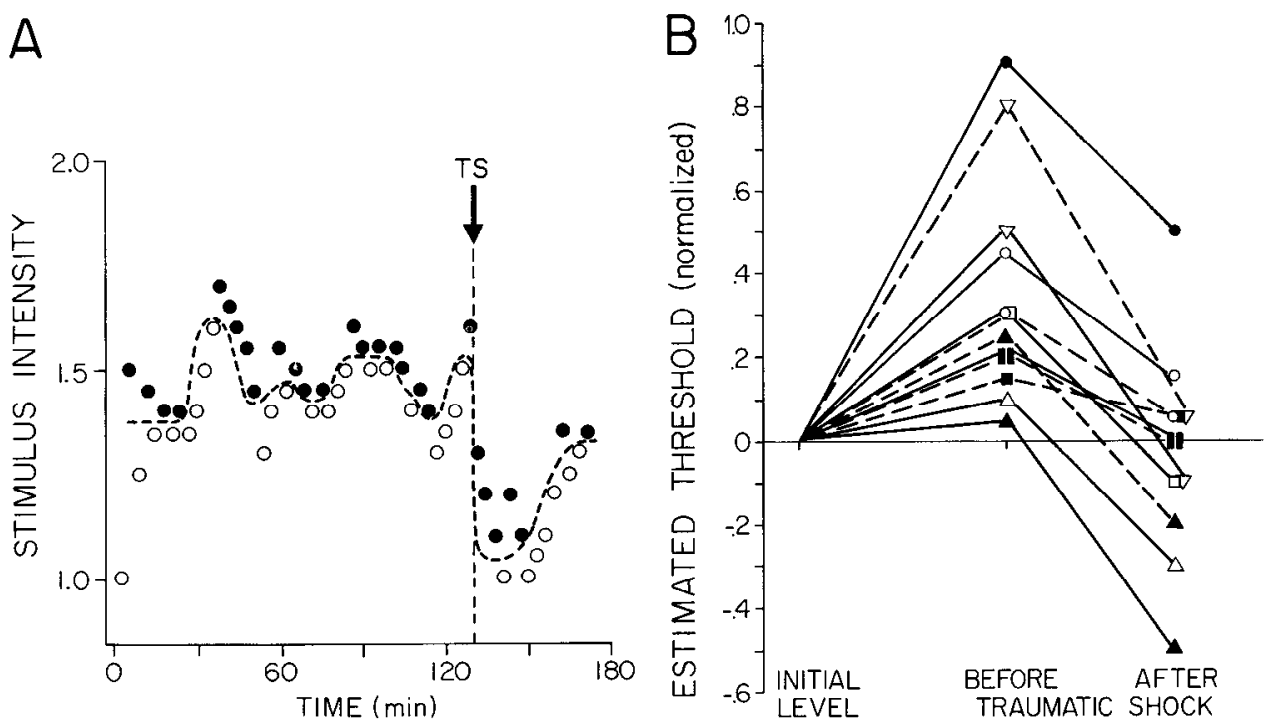

Figure 2. Effect of single traumatic shocks. $A$, Graphic portrayal of a single experimental session. Each trial is portrayed as a point whose abscissa is the time of the trial and ordinate is the strength of test stimulus delivered. Trials on which LG did not fire are plotted as open circles, while trials on which LG did fire are plotted as filled circles. The dashed line gives a subjective interpretation of the probable stimulus threshold for LG firing as a function of time. At the arrow, a single $10 \mathrm{sec}$ TS was applied near the animal's rostrum. The threshold fell and did not completely recover its initial value before the end of the session. $B$, Results for seven animals tested as above. For each session, the LG threshold for the five trials preceding and following a $10 \mathrm{sec}$ TS minus the threshold at the start of the session are given. The TS was in some cases rostral (-) and others caudal (--). Each animal's results are plotted with a different symbol. planted electrodes were presented once every $3 \mathrm{~min}$ throughout the session, and TSs, where necessary, were given at the midpoint of the interstimulus interval. Stimulus levels were continuously varied so as to maintain a running estimate of the stimulus threshold for the unit under observation (see Results). The first day each animal was run, its threshold was tracked without any TSs being given to acclimate it to the testing condition.

\section{Cord severance}

In some experiments, the abdominal nerve cord was severed between first and second ganglia. This was done in cold Ringer's with care to spare the ventral artery. At least $12 \mathrm{hr}$, usually more, were allowed before further testing.

\section{Results}

The stimulus threshold for producing an LG reaction (hereafter simply "threshold") varies throughout the course of an experimental session due to habituation, uncontrolled onset and offset of inhibitory influences that originate rostral to the abdomen (see Krasne and Wine, 1975), and probably other unknown factors. The effects of possible sensitizing stimuli must bc studied against this somewhat variable background. Relatively natural stimuli such as harassing an animal with a stick or bringing one's hand rapidly up to an aquarium, which sometimes cause non-giant escape reactions, do not produce sensitization reliably. For this reason the cffects of strong AC shocks (TSs) were examined.

\section{Effects of single hand applied TSS}

Bringing a shock electrode near an animal and chasing it with the electrode for $10 \mathrm{sec}$ as it attempts to escape usually produces a drop in $L G$ reaction threshold.

Figure $2 A$ illustrates an experimental session. Threshold was first tracked long enough to assess the baseline excitability of LG escape. During such threshold tracking, the stimulus was increased on trials following failures to respond and decreased on trials following LG reactions in an effort to continuously bracket the threshold as narrowly as possible. In the experiment shown, as in most, the lowest preshock threshold was encountered near the start of the session; in this case, there was not a large amount of within-session habituation, but often there was much more. After about $2 \mathrm{hr}$ of such testing, a $10 \mathrm{sec}$ TS to the head region of the animal was given in the interval between two test stimuli. 'Threshold dropped to the lowest level of the session and then began to recover gradually. Although not evident in the experiment shown in Figure $2 A$, threshold often did not reach its lowest level until two or three trials after the TS.

Seven animals were tested in this way with rostral TS; three were additionally tested with caudal TS. The threshold over the first five trials of these sessions, the five trials preceding shock, and the five trials after the TSs are shown in Figure $2 B$. It is apparent that there is generally some rise of threshold (habituation) prior to the TS and a drop of threshold ( $p<0.02$; twotailed Wilcoxin signed ranks test), sometimes to below the level at the start of the session, immediately after the shock.

In such experiments there is some uncertainty as to the exact position of the threshold because of both variability and the fact that the tracking procedure used merely establishes upper and lower limits on the threshold. In most cases where a single measure of threshold level was nceded for statistical analyses, a value half way between the narrowest available upper and lower limits was entered in the analysis. However, in the present experiment, where the existence of a sensitization effect was first being established, a more conservative procedure was employed: The "before shock" threshold in Figure $2 B$ is an estimate of the threshold's lower limit - the largest stimulus at which and below which there were no responses on any of the five trials. For the initial and postshock measures of threshold we used an estimate of the threshold's upper limit-the smallest stimulus that did cause a response during at least one of the subsequent five trials.

At 30 min after traumatic shock, threshold had on the average risen $49 \%$ (SEM $8.3 \%$ ) of the amount it had dropped. In five animals that were followed longer, average recovery was $90 \%$ complete at $1 \mathrm{hr}$.

\section{Repeated TSs via implanted electrodes: \\ Stability of the sensitization effect}

In an effort to obtain still more robust effects and also to ascertain whether the sensitizing stimulus being employed might ultimately serve as an unconditioned stimulus in classical conditioning experiments, the effect of interposing TSs between test trials over a number of consecutive trials was examined. Figure 3 (upper left corner) shows typical results of such an experiment. Threshold dropped conspicuously when TSs were started, usually remained low as long as shocks were delivered (usually about $1 \mathrm{hr}$ ), and recovered slowly after they were terminated.

For 16 animals tested with repeated shocks, median threshold 


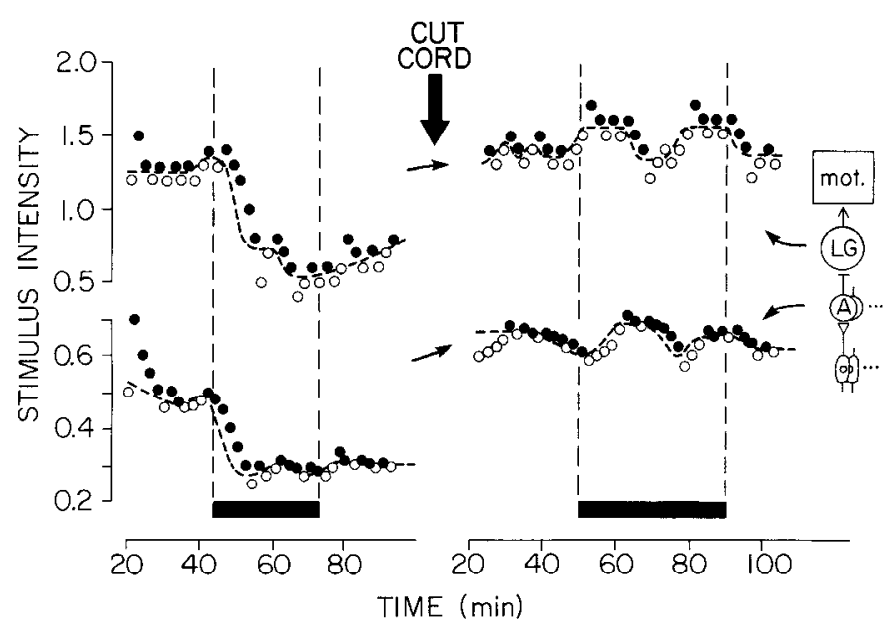

Figure 3. Effect of repeated TSs on threshold of LG and interneuron A before and after severance of the nerve cord. Stimuli were delivered at 2 min intervals with alternate trials used to assess LG (upper graphs) and interneuron A (lower graphs) thresholds. After tracking the threshold for about 45-50 min, $10 \mathrm{sec}$ TSs were delivered to the rostrum 1 min before each trial (black bars on abscissa). In the intact animal (left side), the thresholds of both LG and interneuron A fell during shock and remained lower after discontinuance of shocks. After the nerve cord was severed between the first and second abdominal ganglion, TS no longer caused threshold lowering (right side).

fell from 2.3 shock units (see Materials and Methods) just before initiation of TS trials to 1.6 units at the end of $1 \mathrm{hr}$ of TS. The median drop was 0.92 shock units, and the median drop as a percentage of pre-TS threshold was $40 \%$. Wilcoxin signed ranks tests on both absolute and percentage drops were significant at $p<0.01$ (two-tailed tests). The threshold drops of each animal tested are presented in Figure 4. The threshold of all but three animals (N, O, and P of Fig. 4) was lower after $1 \mathrm{hr}$ of traumatic shocks than just before shock started, despite the fact that many animals had a tendency to habituate to the test stimulus during testing. Of the three animals whose thresholds failed to drop, two (N, O in Fig. 4) did in fact exhibit a decrease during shock, but not enough to compensate for habituation, while one ( $P$ in Fig. 4) increased its threshold during the period in which shocks were given on each of three test sessions, returning to the preshock level shortly after completion of the shock period. Recovery from repeated TSs was $23 \%$ (SEM $4.2 \%$ ) complete at 30 min after cessation of shock ( $n=13$; animals $\mathrm{N}, \mathrm{O}$, and $\mathrm{P}$ of Fig. 4 omitted). In five animals that were followed longer, recovery avcragcd $43 \%$ at $1 \mathrm{hr}$. Recovery always appeared complete after $24 \mathrm{hr}$.

LG reaction thresholds prior to TS varied among animals from 0.85 to 4.0 (which was the maximum stimulus we chose to present). As might be expected from the fact that there is obviously a lower limit to the magnitude of stimulus that can produce a response in even the most "sensitive" animal, animals with initially higher thresholds, which had the potential for dropping greatly, tended to show greater threshold drops to TS than did animals with initially low thresholds (see, e.g., Fig. 8B). It thus seemed desirable to attempt to estimate how far thresholds fell in response to TS, relative to how far they could fall. Since the lowest threshold observed at any time in any experiment was 0.50 stimulus units, we assumed that this level was probably close to the lowest achievable threshold and calculated observed threshold drops as percentages of a "maximal possible" drop to 0.50 stimulus units. Rescaled in this way, the mean percentage drop for repeated traumatic shocks measured at that point during the shock period where threshold was minimal (usually, but not always, the end of the period) was 55\% [SEM

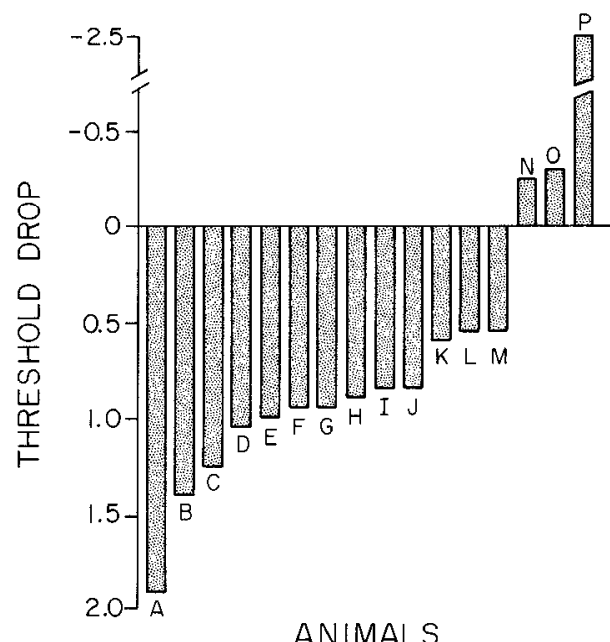

Figure 4. Effect of repeated TSs. The drop in the LG stimulus threshold from the end of the pre-TS period to the end of the TS period is indicated for each animal tested. Individual animals are indexed by letters for text reference.

$6.6 \% ; n=13$; the three animals (see above) not showing a decreased threshold to TS at the end of the TS period were not included in this calculation]. (There was one exception to the observed lowest threshold of 0.50 stimulus units: The threshold of one animal, whose pre-TS threshold of 0.85 was lower than that of any other animal, fell to 0.25 during TS. We presumed that this animal's stimulating electrodes were fortuitously implanted in such a way as to be exceptionally effective, and did not include it in this analysis.)

Multiple TSs appear to be somewhat more effective than single ones. Despite the fact that duration of TSs was commonly less (and never greater) in multiple than single presentation experiments (see Materials and Methods), the mean threshold drop as a percentage of the "maximum possible" drop (computed as above) was $37 \%$ (SEM 5.2\%) for single TS sessions as compared to $55 \%$ (SEM 6.6\%) for multiple TS sessions ( $p<$ 0.06; two-tailed $t$ test), and recovery at $30 \mathrm{~min}$ was $49 \%$ (SEM $8.3 \%$ ) complete for single TS as compared to $23 \%$ (SEM $4.8 \%$ ) for multiple TS sessions ( $p<0.005$; two-tailed $t$ test).

In occasional preparations, TSs of a duration that was usually very effective caused only gradually developing and rapidly recovering sensitization, with recovery sometimes occurring even before TS presentation was discontinued. In such cases, increasing the duration of TS usually led to more typical sensitization results.

Although an effective TS does not readily lose its ability to cause sensitization, after many test sessions it usually does produce somewhat less or less long-lasting sensitization.

\section{Severing neuronal communication with the rostral CNS: Evidence for neurally mediated true sensitization}

Traumatic shocks applied rostrally elevate the excitability of LG reaction circuitry located caudally in the abdomen. The necessary rostrocaudal line of communication could be either neural or systemic. Systemic effects could be due either to a circulating hormone (possibly octopamine) released rostrally and carried caudally in body fluids or to some alteration in circulation or respiration that affects $L G$ reaction excitability. If the route of communication were systemic, sensitization should be producible even after severance of the nerve cord between abdomen and thorax, whereas if the line of communication is neural, sensitization in the abdomen should be abolished after cord section.

Cutting of the nerve cord between abdomen and thorax can 


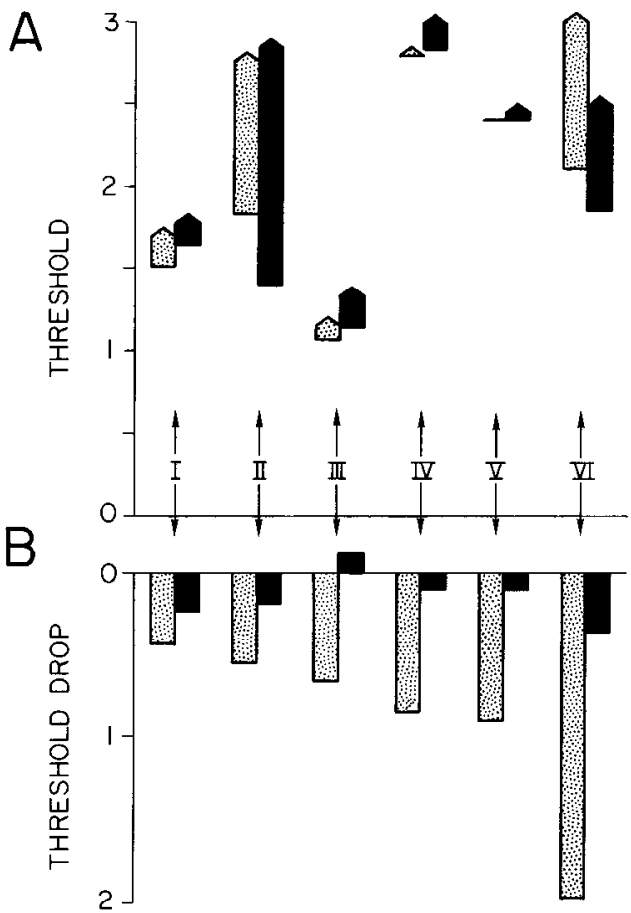

Figure 5. Effect of thoracic-abdominal cord cuts on LG thresholds and ability of rostral shocks to cause sensitization in six animals $(I-V I)$. $A$, Thresholds for each animal prior to shock are shown by pointed bars. Tail of each bar, Threshold at the start of the session; head of bar, Threshold after about $1 \mathrm{hr}$ of threshold tracking just prior to starting TSs. Shaded bars, Sessions on intact animals; dark bars, sessions after cutting the cord. The pre-cut bar for animal $\mathrm{V}$ is a line because there was no habituation during the tracking period. $B$, Drop in threshold produced by $45 \mathrm{~min}-1 \mathrm{hr}$ of TS for the sessions portrayed above. Note that for five of the six animals, when intact, the threshold drop due to sensitization was greater than the rise due to habituation.

also settle another question. It is well established that the LG reaction is subject to inhibition by a pathway that descends into the abdomen from above (Krasne and Wine, 1975). The apparent sensitization effect discussed above might represent a temporary suspension of tonic activity in that descending pathway rather than a true enhancement of excitability. Severance of the cord will remove the effects of any rostrally originating inhibition. If the $L G$ reaction excitability obtained during sensitization in the intact animal is greater than the excitability after removing descending inhibition by cutting the cord, then the sensitization in the intact animal cannot have been due to suspension of inhibition.

Figure 3 (top) illustrates the effects of nerve cord severance. Prior to cutting the cord, clear sensitization was produced by a $10 \mathrm{sec}$ TS. After cutting the cord, the threshold for LG escape dropped slightly but was at all times well above the level seen previously after TS. Moreover, TS no longer had any appreciable effect. Figure 5 summarizes the results for six animals in which comparable experiments were performed. Cutting the cord had little or no effect on threshold prior to TS (Fig. 5A) and always grcatly reduced or abolishcd the ability of traumatic shock to sensitize the reflex (Fig. 5B). Moreover, except for animal II, TS of the intact animal brought threshold below the level seen at any time after cord section.

Since appreciable sensitization no longer occurs after severing the cord, it must depend on neural activity entering the abdomen from above. Since there is little dimunition of threshold simply due to cord section, it may be concluded that the abdominal LG reaction circuitry was not tonically depressed by descending inhibition and therefore sensitization cannot be ascribed to re-

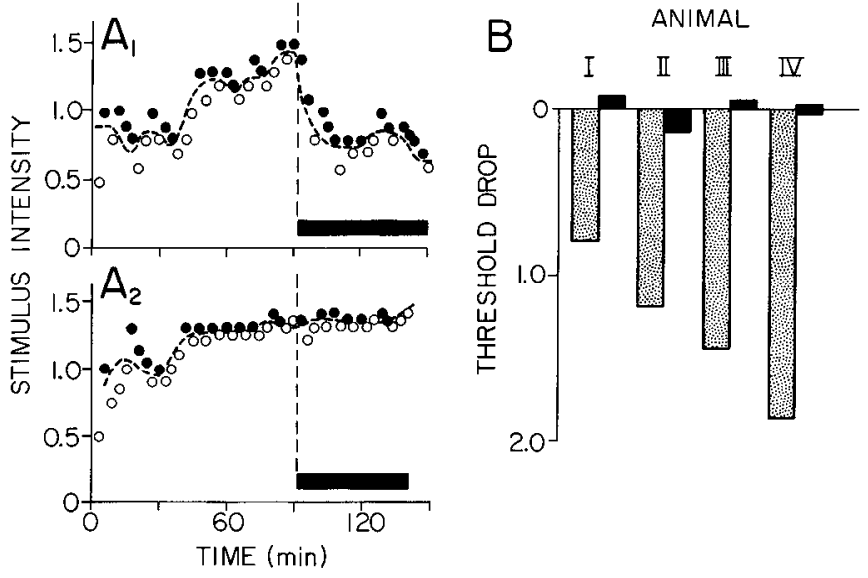

Figure 6. Effect of rostral abdominal cord severance on sensitization to caudal traumatic shocks. $A$, Illustrative sessions before $\left(A_{l}\right)$ and after $A_{2}$ ) cutting cord. Black bar, Period of shock. $B$, Threshold drops in four animals before (shaded bars) and after (black bars) cutting cord.

moval of such inhibition. This conclusion is also reinforced by results on interneuron $A$ reported below.

Although the sensitization effect largely vanishes after cord severance, a small effect sometimes seems to remain. Thus, during the last half-hour of the TS period, two animals of the six whose cord was cut responded consistently to test stimuli slightly weaker than ones they had consistently failed to respond to during the half-hour preceding TS presentation. Although this might have represented chance variation in threshold, it raises the possibility that some systemic (hormonal?) factors contribute to the sensitization effect.

The uniform failure of cord severance to lower pre-TS thresholds in Figure $5 A$ should not be taken to imply that tonic inhibition is never operative under the circumstances of these experiments. About $20 \%$ of the animals we have examined show erratic rises and falls of threshold during testing, presumably due to onset and offset of descending inhibition. We chose not to study such animals further; however, we know from other work that severance of their nerve cords would abolish the swings of excitability and lower their mean thresholds substantially.

\section{Caudal TS: Dependence of sensitization on the rostral CNS}

The previous experiment established that sensitization produced by rostral TSs is produced via an influence that is carried into the abdomen by descending neural pathways. These pathways might be part of the system of neurons that directly causes sensitization, or the sensitization-producing system might in part be intrinsic to the abdomen and be activated by a descending message signaling that a strong noxious stimulus had occurred rostrally. Where the sensitizing system originates and where it can receive activating input therefore requires further clarification.

If the sensitizing system is partly intrinsic to the abdomen, then caudal TSs should be able to produce sensitization even after the nerve cord is severed, whereas if the system originates rostrally and can only be accessed rostrally, cutting the cord should abolish the effects of caudal sensitizing stimuli.

Figure 6 shows the effect of caudal TS before and after cutting the cord between abdomen and thorax. Sensitization is produced by caudal TS in the intact animal, but not in animals in which communication between rostral and caudal halves has been interrupted by severance of the cord between abdomen and thorax. Apparently, the sensitizing system can be accessed only rostrally, and it is its efferent limb that descends into the abdomen. 


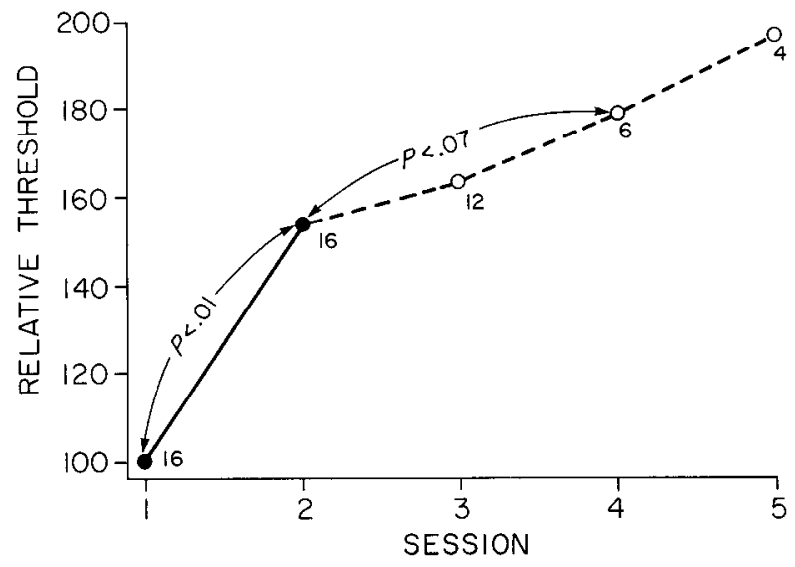

Figure 7. Intersession habituation. The threshold after $1 \mathrm{hr}$ of threshold tracking in five successive daily experimental sessions is graphed as a percentage of the threshold in session 1 . The number of animals tested on each day, indicated next to each point, fell over days because not all animals were tested under suitable conditions for a full $5 \mathrm{~d}$. Statistical significance of threshold differences between days 1 and 2 and 2 and 4 , evaluated by two-tailed Wilcoxin signed ranks tests, are shown.

\section{Sensitization of interneuron $A$ : The first synapse target of descending sensitizing influences}

In Aplysia, sensitization is produced in large measure by facilitation of transmitter release from primary afferents that synapse directly on motor neurons. Decreased release of transmitter from these same afferents is the cause of habituation. The crayfish LG reaction circuit differs from the Aplysia protective withdrawal circuit in having a considerably larger number of serial synaptic relay steps; however, as in Aplysia, habituation appears to be the result of decreased release at the first synapse. Interneuron $A$, the largest of the first-order interneurons that are interposed between afferents and LGs, can easily be recorded in chronic preparations of the sort in use here. Examination of the effects of TS on the stimulus threshold of this neuron can ascertain whether sensitization operates at the primary afferent synapses. Figure 3 (left) illustrates an experiment in which the threshold for both LG and interneuron A were followed by examining each on alternate trials. The experiment is typical of several that were run in showing roughly parallel sensitization effects on the two neurons. Effect of TS on interneuron A thresholds was examined in six animals. Threshold fell in all but one of them, the mean threshold falling from 0.61 stimulus units (SEM 0.18) just before TS to 0.27 units (SEM 0.13) at the end of $1 \mathrm{hr}$ of TS ( $p<0.05$; two-tailed Wilcoxin test). It should also be noted that since interneuron $A$ is not subject to descending inhibition (Krasne and Wine, 1975), this result confirms that sensitization is not due to suspension of a descending tonic inhibition. As with the $L G$, the sensitization of interneuron $A$ can no longer be obtained after severing the cord (Fig. 3, lower half).

\section{Dishabituation or sensitization?}

It has been demonstrated above that the sensitization seen here is not ascribable to removal of any known source of inhibition. However, this still leaves open the possiblity that sensitization mainly serves to restore excitability that has been lost by habituation and that sensitization would be absent or minimal in a fully unhabituated $L G$ reaction.

It is obvious from the data of Figures 2 and 5 that TS does more than restore excitability lost during the stimulation of a single experimental session, because excitability after TS is often greater than excitability at the start of the session. However, as shown in Figure 7, the LG reaction is subject to habituation that lasts between sessions. It therefore remains possible that
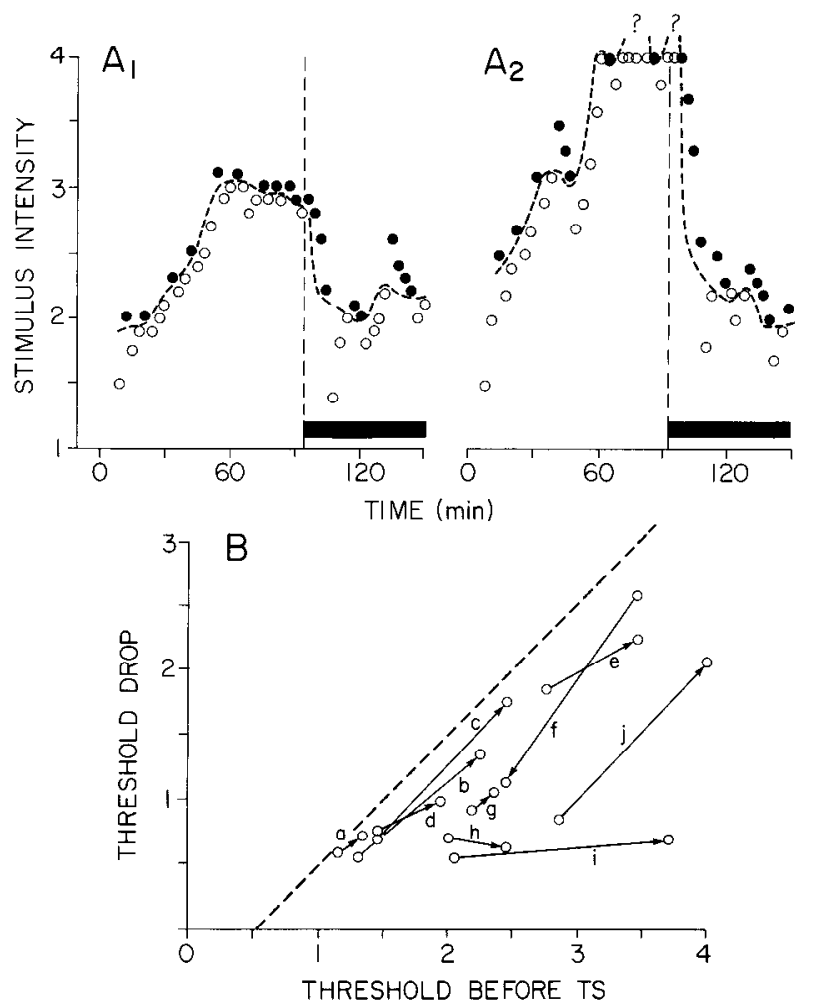

Figure 8. Interactions between sensitization and habituation. $A$, Two consecutive sessions ( $24 \mathrm{hr}$ apart) for a single animal. The threshold just prior to shock trial onset was much higher in the second session because of habituation lasting between sessions. However, during shock trials, the threshold fell to about the same level in both sessions; thus, the threshold $d r o p$ was increased by habituation. $B$, Thresholds before TS (abscissa) and threshold drops due to TS (ordinate) in 10 animals that were tested under comparable conditions on two consecutive sessions. Note that $X$ and $Y$ often increased proportionately. Dashed line, The size of a drop to 0.5 stimulus units, which appears to be the maximum drop possible with the measurement procedures used here. Question marks indicate that threshold was an unknown amount above 4.0 (we chose not to deliver test stimuli greater than 4.0 to avoid possible nerve damage).

TS merely causes recovery from habituation, some of which was established in prior sessions or even prior to an animal's sojourn in the laboratory.

If this were the case, it might be expected that as a given animal's reflex habituates from one session to the next, progressively larger threshold drops should be producible by TS. In the present series of experiments there were 10 cases in which the effects of comparable TSs were tested twice. Figure 8 (top) shows the results for one such animal. Before-shock threshold increased from one day to the next, whereas the postshock threshold was similar on both days; therefore, the amount of sensitization (measured as threshold change) increased markedly as the animal habituated. Figure $8 B$ presents the results for all 10 relevant cases as points on an $X-Y$ plot, with $X$ being the threshold before TS and $Y$ the drop in threshold produced by the TS. The points for different sessions in a given animal are joined by an arrow whose direction indicates the order of the test sessions. On such a plot, data like that in Figure 8A, in which post-TS thresholds are constant from session to session while preshock thresholds rise from one session to the next, are represented by an arrow pointing from left to right with a $45^{\circ}$ slope. The figure shows that in 9 out of the 10 cases available, the threshold drop increased when the preshock threshold increased.

These are the kind of results one might expect if TS simply 
caused a recovery from long-term habituation - the more habituation, the greater the potential for a drop. However, since threshold can apparently not fall below 0.5 stimulus units (see above), there is an effective "floor" to how far thresholds can drop (indicated by the dashed line in Fig. $8 B$ ), and it is possible that near this floor the amount of threshold drop may not fully reflect increases in efficacy of synaptic transmission within the reflex pathway. Thus, an animal with a very powerful sensitization effect that always responded after shock at 0.5 stimulus units might appear to sensitize more as it habituated only because habituation pulled threshold away from its floor. Animals a-d in Figure $8 B$ may well fall into this category, whereas animal $\mathrm{j}$ appears not to do so. The remaining cases are more ambiguous.

Another factor that must be considered in interpreting Figure 8 is that not only the LG reflex but also the effects of TS habituate somewhat from session to session. This will tend to cause $Y$ to drop from session to session independently of changes in $X$ and will work against obtaining increased sensitization as the escape reflex habituates.

\section{Discussion}

The results of this investigation establish that strong stimuli can sensitize escape reactions mediated by the LG neurons of the crayfish nerve cord. This sensitization depends on fiber pathways that descend from above into the abdomen, where the circuitry responsible for exciting the LG cells lies.

The chemically mediated synaptic connections between primary afferents and first-order interneurons are at least one target of the sensitizing influence. Use-induced depression of transmitter release from the presynaptic side of these same synapses is responsible for habituation in crayfish (Krasne, 1976; Zucker, 1972b). And both sensitization and habituation in Aplysia depend on altered transmitter release from the presynaptic side of the corresponding synapses of that animal (Kandel and Schwartz, 1982). In crayfish, it remains to be determined whether it is the pre- or postsynaptic neuron that is affected during sensitization. However, the exaggeration of sensitization in more habituated animals, if real and not simply the result of a threshold "floor" (see above), suggests that the presynaptic terminal, which is the locus of the change responsible for habituation, is involved. It also remains to be determined whether transmission is also altered at other synapses of the LG escape reaction circuit. We have noticed that phasic flexor muscle potentials often increase dramatically during sensitization, but this might well be due to neuromuscular facilitation (Kennedy and Takeda, 1965) produced by repeated usage of the flexor muscle junctions during tail-flipping that occurs during TS.

Octopamine perfused into the isolated abdominal nerve cord mimics the effects seen here (Glanzman and Krasne, 1983). Since octopamine is endogenous in the lobster nervous system (Kravitz et al., 1980), and, probably, also in the crayfish, it is possible that the effects reported here are caused by natural release of octopamine in response to TS. It is consistent with this possibility that both physiological and comparative pharmacological evidence suggests that octopamine in arthropods plays roles similar to those of epinephrine in vertebrates (Kravitz et al., 1980; Robertson and Juorio, 1977). Examination of the effects on sensitization of pharmacological agents that block the effects of octopamine are obviously in order. [Preliminary experiments suggest that phentolamine, a competitive inhibitor of octopamine receptors (Evans, 1981), may interfere with sensitization.] Such work should help determine whether an octopaminergic facilitating system in crayfish plays the same sort of role that has been ascribed to a serotonergic facilitatory system in Aplysia (see Kandel and Schwartz, 1982).

The very long survival of sensitization in the absence of further sensitizing stimuli requires investigation. In animals that have received repeated sensitizing stimuli, the average time to half-recovery was $1 \mathrm{hr}$, and in some preparations there was very little decay of the effect even after $2 \mathrm{hr}$, though recovery always seemed complete after $24 \mathrm{hr}$. The first question that will have to be answered is whether persistence of the effect is due to sustained activity of the descending facilitatory system, local retention of active sensitizing transmitter (octopamine?), or prolonged alteration of the metabolism of the target cells themselves. It is pertinent to note that the enhanced LG reaction excitability caused by exogenously applied octopamine typically decays with a half-time of about $10 \mathrm{~min}$ after drug washout (Glanzman and Krasne, 1983), but even if it were accepted that octopamine mediates sensitization, quantitative comparisons between the behavioral and pharmacological experiments are problematical. In Aplysia, the heterosynaptic facilitation responsible for sensitization is also quite persistent, commonly lasting $15 \mathrm{~min}$ or more. There, it is believed that the persistence is due to a long-lasting activation of presynaptic terminal membrane adenylate cyclase (Schwartz et al., 1983).

The potential for substantial sensitization in this system means that an animal whose LG escape reaction to some stimulus has habituated can, in the face of traumatic events, regain its responsiveness. This is of obvious adaptive value. We believe that sensitization effects have not been seen previously in this system because most experiments have been done on isolated abdomens, on animals whose nerve cords were severed between abdomen and thorax, or on intact animals that were restrained in such a way that descending inhibitory influences turned on by restraint (Krasne and Wine, 1975) masked such sensitization as may have occurred.

\section{References}

Carew, T. J., R. D. Hawkins, and E. R. Kandel (1983) Differential classical conditioning of a defensive withdrawal reflex in Aplysia californica. Science 219: 397-400.

Carew, T. J., E. T. Walters, and E. R. Kandel (1981) Classical conditioning in a simple withdrawal reflex in Aplysia californica. J. Neurosci. 1 : 1426-1437.

Evans, P. D. (1981) Multiple receptor types for octopamine in the locust. J. Physiol. (Lond.) 318: 99-122.

Glanzman, D. L., and F. B. Krasne (1983) Serotonin and octopamine have opposite modulatory effects on the crayfish's LG escape reaction. J. Neurosci. 3: 2263-2269.

Groves, P. M., and R. F. Thompson (1970) Habituation: A dual process theory. Psychol. Rev. 77: 419-450.

Hawkins, R. D., T. W. Abrams, T. J. Carew, and E. R. Kandel (1983) A cellular mechanism of classical conditioning in Aplysia: Activitydependent amplification of presynaptic facilitation. Science 219:400405.

Kandel, E. R., and J. H. Schwartz (1982) Molecular biology of learning: Modulation of transmitter release. Science 218: 433-443.

Kennedy, D. (1971) Crayfish interneurons. Physiologist 14: 5-30.

Kennedy, D., and K. Takeda (1965) Reflex control of abdominal flexor muscles in the crayfish. I. The twitch system. J. Exp. Biol. 43: $211-$ 227.

Klein, M., E. Shapiro, and E. R. Kandel (1980) Synaptic plasticity and the modulation of the $\mathrm{Ca}^{2+}$ current. J. Exp. Biol. 89: 117-157.

Krasne, F. B. (1969) Excitation and habituation of the crayfish escape reflex: The depolarizing response in lateral giant fibers of the isolated abdomen. J. Exp. Biol. 50: 29-46.

Krasne, F. B. (1976) Invertebrate systems as a means of gaining insight into the nature of learning and memory. In Neural Mechanisms of Learning and Memory, M. R. Rosenzweig and E. L. Bennett, eds., pp. 401-429, MIT Press, Cambridge, MA.

Krasne, F. B., and J. J. Wine (1975) Extrinsic modulation of crayfish escape behavior. J. Exp. Biol. 63: 433-450.

Kravitz, E. A., S. Glusman, R. Harris-Warrick, M. Livingstone, T. Schwarz, and F. Goy (1980) Amines and a peptide as neurohormones in lobsters: Actions on neuromuscular preparations and preliminary behavioural studies. J. Exp. Biol. 89: 159-175.

Robertson, H. A., and A. V. Juorio (1977) Octopaminc and somc related noncatecholic amines in invertebrate nervous systems. Int. Rev. Neurobiol. 19: 173-224. 
Schwartz, J. H., L. Bernier, V. F. Castellucci, M. Palazzolo, T. Saitoh, A. Stapleton, and E. R. Kandel (1983) What molecular steps detcrmine the time course of the memory for short-term sensitization in Aplysia? Cold Spring Harbor Symp. Quant. Biol. 48: 811-819.

Sigvardt, K. A., G. Hagiwara, and J. J. Wine (1982) Mechanosensory integration in the crayfish abdominal nervous system: Structural and physiological differences between interneurons with single and multiple spike initiating sites. J. Comp. Physiol. 148: 143-157.

Thompson, R. F., and W. A. Spencer (1966) Habituation: A model phenomenon for the study of neuronal substrates of behavior. Psychol. Rev. 173: 16-43.

Walters, E. T., and J. H. Byrne (1983) Associative conditioning of single sensory neurons suggests a cellular mechanism for learning. Science 219: 405-408.

Wine, J. J., F. B. Krasne, and L. Chen (1975) Habituation and inhibition of the crayfish lateral giant fiber escape response. J. Exp. Biol. 62: 771-782.

Zucker, R. S. (1972a) Crayfish escape behavior and central synapses. I. Neural circuit exciting lateral giant fiber. J. Neurophysiol. 35: 599651.

Zucker, R. S. (1972b) Craytish escape behavior and central synapses. II. Physiological mechanisms underlying behavioral habituation. J. Neurophysiol. 35: 599-651. 